\title{
Multiplex Communication in 2D Mesh Neural Network
}

\author{
T. Kamimura, Y. Yagi \\ ISIR, Osaka University, 8-1 Mihogaoka \\ Ibaraki City, Osaka, 567-0047 Japan
}

\author{
S. Tamura \\ NBL Technovator Co., Ltd. 631 Shindachimakino \\ Sennan City, 590-0522 Japan
}

\author{
Y.W. Chen \\ Ritsumeikan University 1-1-1 Nojihigashi, Kusatsu-shi \\ Shiga, 525-8577 Japan
}

\begin{abstract}
We have already analyzed and reported on multiplex communication in neural network. However, these are composed and designed so as to force the network communicate with multiplexed manner using "code" or "temporal sequence." That is, the network has a main loop and some coding/decoding circuits, and these are somewhat artificial. In this paper, we show without these artificial guidance, it is also possible to communicate with multiplexing in a natural 2D grid shape neural network, where some learning algorithm are employed to facilitate to find paths from a transmitting neuron to a receiving neuron. We also show that in these neural networks, random sequences are more frequent in number than that of non-random sequences.
\end{abstract}

Keywords-brain information processing; neural circuit; pseudo random sequence; $M$-sequence; multiplex communication

\section{INTRODUCTION}

We have already proposed a multiplexed communication model on a 2D neural network which is composed of a main loop circuit and some coding/decoding neural networks [1]. The network is forced to communicate with multiplexed manner, since between three transmitting neurons and three receiving neurons of three communication channels, there are only two lines. Then it was observed that the network generates and uses pseudo random sequences including M-sequences frequently. It is well known that in many artificial real communication systems, e.g., mobile phones, pseudo random sequences, especially M-sequences, are employed as almost orthogonal codes which are easy to discriminate each other. Further, we have observed M-sequence family in spike trains from cultured neural networks significantly more than by chance $[2,3]$.

A problem remains whether it is possible to communicate multiplexing manner in more natural neural networks without such artificial shape to force multiplexing. Therefore, we treat in this paper a more natural homogeneous grid shape neural network.

\section{PATH SEARCH MODEL}

It is well known that number of synapses of a neuronal cell is more than 1000, and therefore there exist a large number of connection paths as well as loops in a neural network [4-6]. To a neuronal cell, stimulation is transmitted from other neuronal cell through synapses, and membrane electrical potential is increased. If the electrical potential exceeds a threshold, the neuronal cell is fired, and if under the threshold, it is not fired. Firing model of the neuronal cell is shown in Eq.(1).

$$
E(t)= \begin{cases}1 & \sum_{n=0}^{N} E_{n}(t-1) W_{E, E_{n}}>\text { threshold } \\ 0 & \text { otherwise }\end{cases}
$$

Here, $E(t)$ represents a state of cell $E$ at time $t, 1$ represents firing state, and 0 represents static state. $N$ is number of other cells connected to the cell, and $W_{E, E n}$ is connection weight between $E$ and $E_{n}$. That is, the state of a cell is determined with whether the total sum of the products of connection weight and state at the one clock previous time of other cell connected to the cell exceeds a threshold or not. As for each neuron cell, a state will change by progress of $t$. We regard the communication is succeeded if an output cell is fired when an external stimulation is given to an input neuron cell and the stimulation is transmitted to the output cell through neuron cells in the neural network.

We made a multiplexing communication model of $2 \times 2$ with 2 input neuron cells for transmitting the information and corresponding 2 receiving neuron cells on a $9 \times 9$ grid shape neural network. We call this "path search model." It is shown in Figure 1(a). Here, $\alpha, \beta$ are input cells for transmitting the information, and $\alpha^{\prime}, \beta^{\prime}$ are cells for receiving information $\alpha, \beta$, respectively. Between a cell and its 8 neighbouring cells, connection weights are set randomly at time 0 within $[-1,1]$. Firing information input to the input cells $\alpha, \beta$ is transmitted in the network with the transition of the states of cells according to the connection weights set between cells and Eq.(1) by progress of time step.

Further, cell happens to be fired sometimes voluntarily and randomly irrespective of the transition of the firing. We call this "random firing." With the firing by the transition of state and random firing, firing cells go moving.

Example of the state transition is shown in Figure 1. We can see that firing of $\alpha$ in Figure 1(a) at time $t=0$ is changed to Figure $1(\mathrm{~b})$ at time $t=1$. 


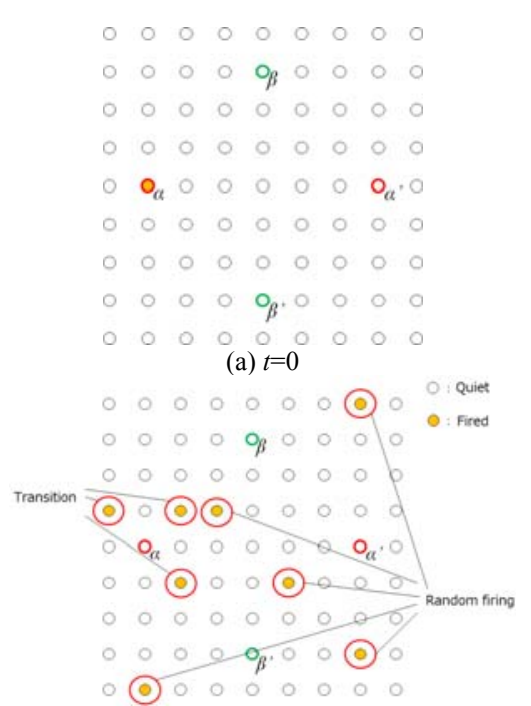

(b) $\mathrm{t}=1$

FIGURE I. EXAMPLE OF STATE TRANSITION.

\section{A. Updating Weights}

Weight set between cells expresses transmission efficiency of synapse. Synapse transmission efficiency in natural neuronal network is reinforced if post synaptic neuron is fired by firing of presynaptic neuron. This is called Hebb rule. Also in the path search model, connection weights are updated based on the Hebb rule. In this case, there are two cases of the weight updating rule of reinforce by simultaneous firing and that of by one clock before firing. These are expressed by Eq.(2) and Eq.(3).

$$
\begin{aligned}
& \Delta w_{i}=\alpha s_{i}(t) s_{i+1}(t)+\beta_{1} w_{i-1}+\beta_{2} w_{i+1} \\
& \Delta w_{i}=\alpha s_{i}(t) s_{i+1}(t-1)+\beta_{1} w_{i-1}+\beta_{2} w_{i+1}
\end{aligned}
$$

Here, $w_{i}$ is attention paid weight, $s_{i}(t)$ is state of cell $i$ at time $t$, and $i+1$ represents cell which is connected to cell $i$ with weight $w_{i}$. Weights $w_{i-1}$ and $w_{i+1}$ represent weights ahead of extending weight along the connected direction, and have a role of inertia term to make the weight update smoothly. Further, $\alpha, \beta_{1}, \beta_{2}$ are respective coefficient parameters.

In the case of simultaneous firing reinforcement, as shown in Eq.(2), the weights are updated by referring states of cell $i$ and cell $i+1$ at time $t$, and in the case of firing reinforcement at one clock time before, weights are updated by referring state of cell $i$ at time $t$ and state of cell $i+1$ at time $t-1$, respectively. Increment of $\Delta w_{i}$ by weight update is divided equally to remained neighbours, and each weights in neighbour are deducted as much. Therefore, total sum of weights of whole model is not changed even after the weights are updated.

\section{B. Success or Failure of Communication}

In succession of the state transition, there becomes a case where the firing information of transmitting side $\alpha, \beta$ is transmitted to the receiving side $\alpha^{\prime}, \beta^{\prime}$ and they are fired. In this case, we can regard that the information is transmitted and we regard the communication was succeeded.
In case of $2 \times 2$ multiplex communication, information from $\alpha$ should be transferred to $\alpha^{\prime}$, and that of $\beta$ should be to $\beta$ '. Therefore, when the information is input to $\alpha$ at $t=0$, number of firing between $t=6$ to $t=63$ should be $\alpha^{\prime}>\beta$, and when the information is input to $\beta$ at $t=0$, number of firing of $\beta$ ' should be $\alpha^{\prime}<\beta$ ', and only in such case, we regard the communication became succeeded fully.

\section{Sequence Appeared at Cell}

When we observe the whole 81 cells of the path search model and pay attention to each cell, we can obtain a sequence in which 0 and 1 appear by progress of time step $t$. Then we can obtain knowledge what contribute to $2 \times 2$ communication by paying attention to and analysing these sequences.

\section{EXPERIMENT AND DISCUSSION}

We did simulation experiment of $2 \times 2$ communication by using the path search model.

\section{A. Experiment 1}

We performed the experiment by 3 steps of the weight learning term, an $\alpha$ test, and a $\beta$ test.

Weight learning term: We perform up to $t=63$ path search with setting a state of $\alpha$ of Figure 1 as 1 at $t=0$. In other words, we update the connection weights based on the leaning rule.

$\alpha$ test: Using a model after the weight learning, we make a state of $\alpha$ of Figure 1 as 1 at the time of $t=0$ and watch the state transition up to $t=63$. At this process we do not update the weights. Here, if $\alpha^{\prime}>\beta$ ', $\alpha$ test is success, and if $\beta^{\prime}<\alpha^{\prime}$, we regard $\alpha$ test is failure.

$\beta$ test: Using a model after the weight learning, we make a state of $\beta$ of Figure 1 as 1 at the time of $t=0$ and watch the state transition to $t=63$. At this process we do not update the weights. Here, if $\beta^{\prime}>\alpha^{\prime}, \beta$ test is success, and if $\alpha^{\prime}<\beta^{\prime}$, we regard $\beta$ test is failure.

\section{B. Experiment 2}

In a weight learning term, we performed weights were updated only by transition from $\alpha$ in Experiment 1 . Therefore we performed weight update only by information of the transmission side and did not have the information of the cell of the reception side that was a goal of the information. Therefore, in Experiment 2, we made both of the states of $\alpha$ and $\alpha^{\prime}$ as 1 at time $t=0$ and performed path search up to $t=63$.

\section{Experiment 3}

In the weight learning of Experiment 1 and 2, the learning was made only based on information of the $\alpha$ side and did not have the information of the $\beta$ side. Therefore, in Experiment 3, we made both of the states of $\alpha$ and $\alpha^{\prime}$ as 1 at time $t=0$ and performed the path search up to $t=31$. After then, we cleared all the states and made states of $\beta$ and $\beta^{\prime}$ as 1 at time $t=0$ and performed path search up to $t=31$.

We show results of Experiment 1, 2, and 3 are shown in Figure 2. Here, the full success means the case where both of $\alpha$ test and $\beta$ test succeeded. From each experiment, we can see that in the path search model, $2 \times 2$ communication can be realized from randomly given initial weights. 
In addition, learning becomes easy to advance by using not only the information of the transmission side but also the information of the reception side and we see that the number of the success increases from comparison of Experiments 1 and 2. And we know that the number of the success of $2 \times 2$ communication increases by doing learning from both of $\alpha$ and $\beta$ sequentially not pushing forward learning only from either of transmitting cells, from Experiment 3.

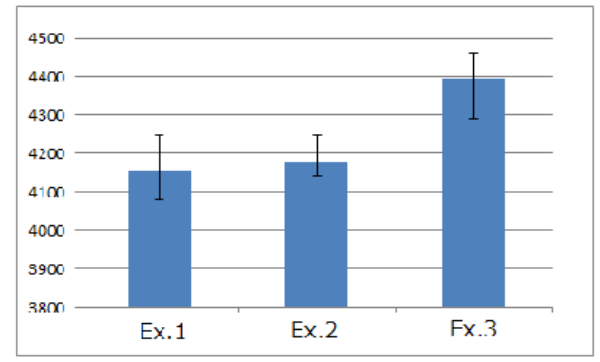

FIGURE II. AVERAGE NUMBER OF FULL SUCCESS AMONG 106 TRIALS. EACH EXPERIMENT IS REPEATED 5 TIMES AND ITS STANDARD DEVIATION IS ALSO SHOWN AS ERROR BAR.

\section{Experiment 4}

In Experiment 3, by changing the parameters of random firing rate, the firing threshold of the cell, reinforcement by simultaneous firing or reinforcement by 1 clock time before firing, we observed what kind of sequence appeared more often when the $2 \times 2$ communication was successful or failed. Parameters we varied was two kinds of 0.1 and 0.05 of the random firing rate, 4 kinds of $0,0.25,0.5$, and 0.6 of firing threshold, and two kinds of reinforcement by simultaneous firing or reinforcement by 1 clock time before firing.

The target sequences to be checked are ones surrounded with 1 at both ends and whose length is 3 to 8 . That is, they are totally 120 kinds of

Length 3: [ $\left.\begin{array}{lll}1 & 1 & 1\end{array}\right]$ of one kind

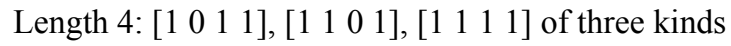

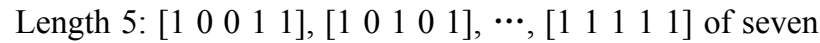
kinds

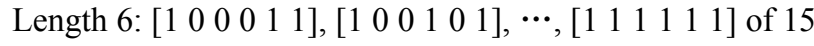
kinds

Length 7: [1 $\left.\begin{array}{lllllll}1 & 0 & 0 & 0 & 0 & 1 & 1\end{array}\right],\left[\begin{array}{lllllll}1 & 0 & 0 & 0 & 1 & 0 & 1\end{array}\right], \cdots,\left[\begin{array}{lllllll}1 & 1 & 1 & 1 & 1 & 1 & 1\end{array}\right]$ of 31 kinds

Length 8: [1 $\left.\begin{array}{llllllll}1 & 0 & 0 & 0 & 0 & 0 & 1 & 1\end{array}\right],\left[\begin{array}{llllllll}1 & 0 & 0 & 0 & 0 & 1 & 0 & 1\end{array}\right], \cdots,\left[\begin{array}{lllll}1 & 1 & 1 & 1 & 1\end{array}\right.$ $111]$ of 63 kinds.

We classify these 120 kinds of sequences into two kinds of ones whose arrangement of 0,1 is random like and one not. That is;

Case (1): we regard as non-random sequences 6 kinds

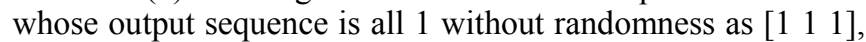
$\left[\begin{array}{llll}1 & 1 & 1 & 1\end{array}\right], \ldots,\left[\begin{array}{lllllllll}1 & 1 & 1 & 1 & 1 & 1 & 1 & 1\end{array}\right]$, and others as random sequences.
Case (2): we regard as non-random sequences in addition

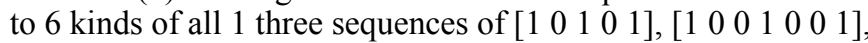
$\left[\begin{array}{lllllll}1 & 0 & 1 & 0 & 1 & 0 & 1\end{array}\right]$.

For these output sequences, we compare the number of the appearances when the communication was succeeded and failed. We divide the number of the appearances when succeeded by the number of the appearances when failed. In other words, if these values exceed 1, the sequence is relatively frequent when communication succeeded, and if less than 1, the sequence is relatively frequent when communication failed. We show a part of the averages of rates of detected sequences for all parameters in Figure 3.

From Figure 3, we can see that perfectly non-random sequences of [111], [1111], [11111] of all 1 output became less than 1, and others became to exceed 1 . This result shows that when the $2 \times 2$ communication succeeded, random like sequences appeared relatively frequently, and when failed, non-random like sequences appeared relatively frequently. Then, we think that random like sequences contribute to the success of the communication.

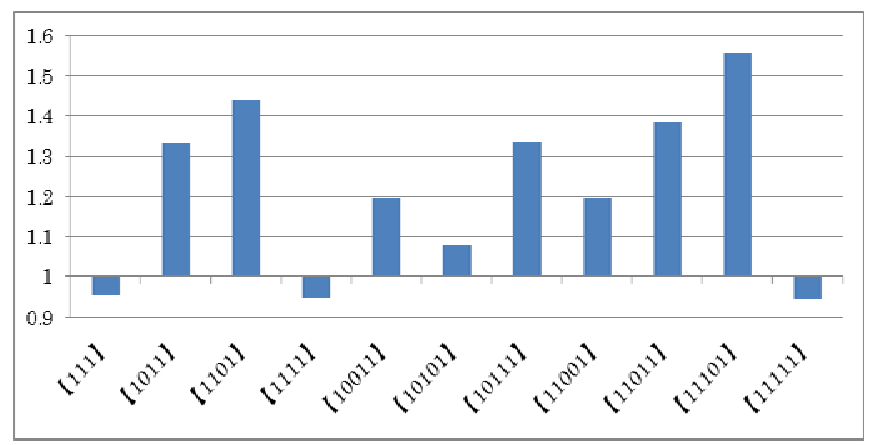

FIGURE III. A PART OF RATES OF DETECTED SEQUENCE.

In Figure 4, output ratios of whole sequences, random sequences, and non-random sequences with changing the threshold are shown. In this figure, the non-random sequences mean those of all 1. We can see from Figure 4 that irrespective of firing threshold, random sequences appear more when the communication succeeded than compared with when failed.

In Figure 5, result of regarding 9 kinds including 3 kinds of $\left[\begin{array}{lllll}1 & 0 & 1 & 0 & 1\end{array}\right],\left[\begin{array}{lllllll}1 & 0 & 0 & 1 & 0 & 0 & 1\end{array}\right],\left[\begin{array}{lllllll}1 & 0 & 1 & 0 & 1 & 0 & 1\end{array}\right]$ as non-random sequence is shown.

In addition, we know that a difference of the number of the output random sequences and non-random sequences in case of communication success and the failure when increasing the threshold from Figure 5. Because this is that the firing threshold of the cell rises, and quantity of firing decreases, and a degree of difficulty of the information and communication went up, and it is thought that random characteristics of the sequences became more important.

The matter that is important to the success or failure of the communication knows that it is random characteristics of a sequence output by a cell in a path search model as a result of the experiments. 


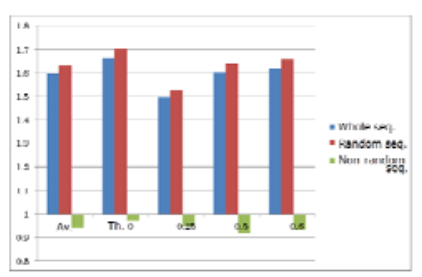

FIGURE IV. COMPARISON OF OUTPUT CHANGING THRESHOLD. NON-SEQUENCES MEAN THOSE OF ALL 1

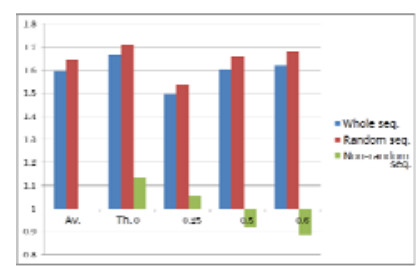

FIGURE V. SEQUENCES FOR COMPARIS RANDOM

\section{CONCLUSION}

By this study, we built a path search model, and, in the model, it was shown that $2 \times 2$ multiplex communication was possible. In addition, in the possibility of the multiplex communication, we knew that the random nature of the sequence of pulse output by a cell had an important meaning. Specifically, we often failed in multiplexing communication when the network was observed to has many cells which cause less-random firing phenomena, and on the contrary, we often succeeded in multiplexing communication when the network was observed to has many cells which cause rich random firing phenomena

From these, we knew that random sequence or strongly random sequence participated in the possibility of the communication deeply in time and space multiplexing communication.

\section{REFERENCES}

[1] S. Tamura, Y. Nishitani, T. Kamimura, et al. "Multiplexed Spatiotemporal Communication Model in Artificial Neural Networks," Automation, Contr. and Intell. Syst. Vol. 1, No. 6, 2013, pp. 121-130. doi: 10.11648/j.acis.20130106.11.

[2] Y. Nishitani, C. Hosokawa, Y. Mizuno-Matsumoto, T. Miyoshi, H. Sawai and S. Tamura, "Detection of M-sequences from spike sequence in neuronal networks," Computational Intelligence and Neuroscience Volume 2012, 2012.

[3] S. Tamura, Y. Nishitani, C. Hosokawa, et al., "M-sequence family from cultured neural circuits," ISSDM 2012, Oct. 23-25, 2012

[4] C. Lecerf, "The double loop as a model of a learning neural system," Proc.World Multiconf. on Syst., Cybern. and Informatics, Vol.1, pp. 587594, 1998.

[5] Y. Choe, "Analogical cascade: a theory on the role of the thalamocortical loop in brain function," Neurocomputing 52-54, pp.713-719, 2003.

[6] T. Kamimura, Y. Nishitani, Y.-W. Chen, Y. Yagi, and S. Tamura, "Copy of neural loop circuits for memory and communication," Journal of Communications and Information Sciences, Vol.4, No.1, pp.46-56, Jan 2014. 